\title{
Farthest points on flat surfaces
}

\author{
Joël Rouyer Costin Vîlcu
}

March 28, 2022

\section{Introduction}

This note is an elementary example of the reciprocal influence between Topology and Geometry. A well-known result in this direction is the celebrated GaussBonnet theorem, which easily implies that the only flat Riemannian surfaces are flat tori and flat Klein bottles. (All surfaces considered here are compact and without boundary.) We consider the distance function from an arbitrary point $p$ on such a surface, and are interested in the set $F_{p}$ of all farthest points (i.e., points at maximal distance) from $p$. Denote by $F$ the (multi-valued) mapping thus defined.

Hugo Steinhauss asked for characterizations of the sets of farthest points on convex surfaces, see Section A35 in [2]. In particular, he asked if the spheres are the only convex surfaces for which $F$ is a single-valued involution. A series of several papers, starting with [16, answered Steinhauss' questions. The survey [13], though a little dated, is still a reference in the field. It should be noted that few examples of explicit computations of farthest points are known, see [6] or even [5], and the counterexamples to the mentioned conjecture of Steinhauss [12, [14, 3], 4]. On the other hand, no convex polyhedron has $F$ a single-valued involution [7].

A motivation for this note lies in the application of Baire Categories theorem to the study of farthest points on Alexandrov surfaces, initiated in [15] and continued in 9. Roughly speaking, an Alexandrov surface with curvature bounded below by $\kappa$ is a 2-dimensional topological manifold endowed with an intrinsic metric which verifies Toponogov's comparison property. For the precise definition and basic properties of Alexandrov surfaces, see for example [1, 10] or, closer to our topic, [11. It is easy to see that convex surfaces are examples of Alexandrov surfaces with curvature bounded below by 0 . Conversely, any such Alexandrov space is isometric to a (possibly degenerate) convex surface whenever it is homeomorphic to the sphere.

Consider the space $\mathcal{A}(\kappa)$ of all Alexandrov surfaces, with the topology induced by the Gromov-Hausdorff metric. Two surfaces lie in the same connected component of $\mathcal{A}(\kappa)$ if and only if they are homeomorphic to each other [8]. Hence convex surfaces form a connected component of $\mathcal{A}(0)$.

By a variant of the Gauss-Bonnet theorem, any topological torus or Klein 
bottle in $\mathcal{A}(0)$ is (Riemannian and) flat. So two connected components of $\mathcal{A}(0)$ contain only flat surfaces.

In [9] we extend Tudor Zamfirescu's results in [17, [18, and show that on most (in the sense of Baire categories) Alexandrov surfaces outside the aforementioned components, most points have a unique farthest point. The cases of the connected components of flat surfaces, namely the components of $\mathcal{A}(0)$ containing flat tori and flat Klein bottles respectively, are treated in this note with elementary techniques. Somehow intriguingly, at least at a first glance, flat tori behave differently with this respect from flat Klein bottles, and both classes of flat surfaces behave differently from all other Alexandrov surfaces.

It is well known that a flat surface can be obtained as the quotient of the Euclidean plane $\mathbb{E}$ by a discrete group of isometries $\Lambda$. Alternatively, it can be obtained identifying opposite sides of a parallelogram $P$; the correspondence between those constructions is almost obvious. What is a little less known is that, for Klein bottles, $P$ can be chosen to be a rectangle without loss of generality, while for tori, its side lengths and angles may be asked to satisfy a certain inequality. For aim of completeness, before determining their farthest points, we reprove the classification of flat surfaces.

On a flat surface $S=\mathbb{E} / \Lambda$, the cut locus $C(p)$ of a point $p$ (see any book of Riemannian geometry for the general definition) is the the set of all points in $S$ joined to $p$ by at least two segments (i.e., shortest paths). Hence it is the image under the canonical surjection of the Voronoi diagram of $p$, seen as a set of points in $\mathbb{E}$. In particular, the cut loci on flat surfaces are graphs without extremities, whose edges are segments. Moreover, one can easily see that a farthest point from $p$ is necessarily a vertex (ramification point) of $C(p)$. Recall that the Voronoi diagram of a discrete set of sites (i.e., fixed points) $S \subset \mathbb{E}$ is the union of the boundaries of the cells $V_{s}(s \in S)$ consisting of the points of $\mathbb{E}$ that are closer to $s$ than to any other site in $S$.

We shall denote by $F_{p}^{n}$ the set of farthest points from $p$ which are joined to $p$ by exactly $n$ segments and by $\# S$ the cardinality of the set $S$.

\section{$2 \quad$ Flat tori}

Denote by $T_{a, b, \alpha}$ the torus obtained (by the usual identification) from a parallelogram of side lengths $a$ and $b$ and angle $\left.\alpha \in] 0, \frac{\pi}{2}\right]$ between them.

Theorem 1. Any flat torus is isometric to some $T_{a, b, \alpha}$ with $a, b, \alpha$ satisfying

$$
2 b \cos \alpha \leq a \leq b .
$$

Proof. Let $T$ be a flat torus. The shortest non-contractible closed curve $\gamma_{0}$ is a simple closed geodesic; denote by $a$ its length. For $\varepsilon>0$ small enough, the $\varepsilon$-neighborhood $N_{\varepsilon}$ of $\gamma_{0}$ is a flat cylinder. The boundary of the strip has two connected components, which are simple closed geodesics on $T$ of length $a$. Let $\beta_{\varepsilon}^{0}, \beta_{\varepsilon}^{1}: \mathbb{R} / a \mathbb{Z} \rightarrow K$ be their arc-length parametrizations. Since $T$ has finite area, for greater $\varepsilon$ the strip must self overlap (i.e., Area $\left(N_{\varepsilon}\right)=2 a \varepsilon-$ 
Area (overlaying) $<2 a \varepsilon$ ); let $c$ be the greatest $\varepsilon$ such that it does not. There are distinct points $t_{0}, t_{1} \in \mathbb{R} / a \mathbb{Z}$ such that $\beta_{c}^{u_{0}}\left(t_{0}\right)=\beta_{c}^{u_{1}}\left(t_{1}\right)$, for some $u_{0}, u_{1}$ taking values in $\{0,1\}$. Moreover there exists $s= \pm 1$ such that

$$
\dot{\beta}_{c}^{u_{0}}\left(t_{0}\right)=s \dot{\beta}_{c}^{u_{1}}\left(t_{1}\right)
$$

for otherwise $\beta_{\varepsilon}^{u_{0}}$ and $\beta_{\varepsilon}^{u_{1}}$ would have intersected for lower $\varepsilon<c$.

Assume first that $u_{0}=u_{1}=0$ (the case $u_{0}=u_{1}=1$ is obviously similar). Because of (2), the lowest $\varepsilon$ such that $\beta_{\varepsilon}(t)$ has more that one preimage in $\mathbb{R} / 2 a \mathbb{Z}$ does not depend on $t$ and equals $c$. Moreover, since the running direction of $\beta_{c}^{0}$ cannot change all of sudden, $s=1$. Hence $\beta_{c}^{0}\left(t_{0}+t\right)=\beta_{c}^{0}\left(t_{1}+t\right)$; it follows that $\left|t_{0}-t_{1}\right|=a / 2$ and a neighborhood of $\beta_{c}^{0}$ is isometric to a Möbius band, in contradiction with the fact that $T$ is orientable.

Hence $u_{0} \neq u_{1}$; assume $u_{0}=0, u_{1}=1$ and set $\beta_{-\varepsilon}^{0}=\beta_{\varepsilon}^{1}, \varepsilon \in[0, c]$. One can assume without loss of generality that $\beta_{\varepsilon}^{u}$ is parametrized in such a way that $\tau:[-c, c] \rightarrow T, \varepsilon \mapsto \beta_{\varepsilon}^{0}(0)$ is a geodesic normal to $\beta_{0}^{0}$ and $\dot{\beta}_{\varepsilon}^{0}(0)$ is parallel along $\tau$. By (2), $\beta_{c}^{0}$ and $\beta_{c}^{1}$ are two parametrizations of the same geodesic, that is

$$
\beta_{c}^{1}(t)=\beta_{c}^{0}( \pm t+d) .
$$

Hence $T$ is the cylinder $C \stackrel{\text { def }}{=} \operatorname{Im} \gamma_{0} \times \operatorname{Im} \tau$ whose boundary points are pairwise identified: $\left(\gamma_{0}(t), \tau(-\varepsilon)\right)$ with $\left(\gamma_{0}( \pm t+d), \tau(\varepsilon)\right)$. Since $T$ is orientable, the sign of $t$ has to be plus. Reversing if necessary the running direction of all geodesics $\beta_{\varepsilon}^{u}$, one can assume without loss of generality that $d \in[0, a / 2]$. The line segment of $C$ joining $\beta_{c}^{1}(0)$ and $\beta_{c}^{0}(d)$ provides a close geodesic of $T$ of length $b \stackrel{\text { def }}{=} \sqrt{d^{2}+4 c^{2}}$. Cutting $C$ along this line provides a parallelogram with side lengths $a, b \geq a$ (because $\gamma_{0}$ is a shortest closed geodesic) and angle

$$
\alpha=\arccos \frac{d}{b} \geq \arccos \frac{a}{2 b} \text {. }
$$

Theorem 2. Let $p$ be a point of the flat torus $T_{a, b, \alpha}$ (with $\left.2 b \cos \alpha \leq a \leq b\right)$. If $\alpha=\pi / 2$ then $\# F_{p}=\# F_{p}^{4}=1$, else $\# F_{p}=\# F_{p}^{3}=2$.

Proof. $T_{a, b, \alpha}$ is isometric to the standard plane $\mathbb{R}^{2}$ quotiented by the group of translations generated by the vectors $u \stackrel{\text { def }}{=}(a, 0)$ and $v \stackrel{\text { def }}{=}(b \cos \alpha, b \sin \alpha)$. Let $\phi: \mathbb{R}^{2} \rightarrow T_{a, b, \alpha}$ be the canonical surjection and fix as origin $o=(0,0)$ of $\mathbb{R}^{2}$ a point in $\phi^{-1}(p)$.

Put $S \stackrel{\text { def }}{=}(a, 0) \mathbb{Z}+(b \cos \alpha, b \sin \alpha) \mathbb{Z} \subset \mathbb{R}^{2}$. We have $\phi^{-1}(p)=S$ and the cut locus of $p$ is the image under $\phi$ of the Voronoi diagram of $S$.

If $\alpha=\pi / 2$, the Voronoi diagram is a regular rectangular tiling of the plane, and the conclusion follows immediately. So we may assume, from now on, that $\alpha<\pi / 2$; consequently, the tiles are non-rectangular parallelograms.

We claim that the restriction to a parallelogram $P$ of the Voronoi diagram of $S$ is the same as the Voronoi diagram of the four vertices of $P$. For this, it 


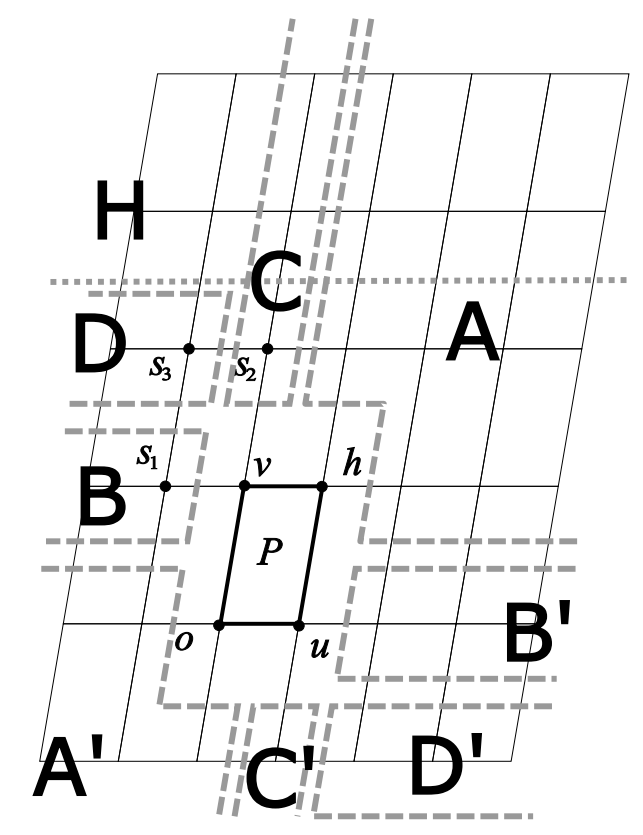

Figure 1: Tiling of the plane generated by a flat torus $T_{a, b, \alpha}$.

is sufficient to prove that for any point $x \in P$ and any $s \in S$ which is not a vertex of $P$, there is a vertex $w$ of $P$ such that $\|s-x\|>\|w-x\|$. Choose, for instance, the parallelogram $P$ whose vertices are $o \stackrel{\text { def }}{=}(0,0), u \stackrel{\text { def }}{=}(a, 0)$, $v \stackrel{\text { def }}{=}(b \cos \alpha, b \sin \alpha)$ and $h \stackrel{\text { def }}{=} u+v$. Put $s_{1} \stackrel{\text { def }}{=} v-u, s_{2} \stackrel{\text { def }}{=} 2 v$ and $s_{3}=2 v-u$.

If $s=m u+n v$ with $m, n \in \mathbb{Z}, m, n \geq 1$ (zone A in Figure 1) then we can choose $w=h$, for the angle $\measuredangle x h s$ is obtuse. Similarly, if $s=m u+n v$ with $m, n$ negative integers (zone $\mathrm{A}^{\prime}$ ) then we can choose $w=o$.

Since, by (1), $b=\|v\|<\|v-u\|, o$ (and hence $P$ ) lies on the right hand side of the bisector of the line-segment $\left[v, s_{1}\right]$. It follows that we can choose $w=v$ for any $s=s_{1}-k u, k \geq 0$ (zone B). By symmetry, one can choose $w=u$ for $s=u+k u, k \geq 1$ (zone $\left.\mathrm{B}^{\prime}\right)$.

Still by (1), $a=\|u\|=\|h-v\|<\left\|h-s_{2}\right\|$, hence $h$ is separated from $s_{2}$ by the mediator $m_{2}$ of the line-segment $\left[s_{2}, v\right]$. It follows that $P$ lies in the lower half-plane bounded by $m_{2}$ and we can choose $w=v$ for any $s=s_{2}+k v, k \geq 0$ (zone C). By symmetry, one can choose $w=u$ for $s=u-k v, k \geq 1$ (zone C'). The condition (1) also implies that $P$ lies on the right hand side of the mediator of $\left[s_{2} s_{3}\right]$, whence one can also choose $w=v$ for any $s=s_{3}-k u, k \geq 0$ (zone D). By symmetry one can chose $w=u$ for $s=k u-v, k \geq 1$ (zone D').

Assume now that $s=k u+k^{\prime} v, k^{\prime} \geq 3, k \in \mathbb{Z}$ (zone $\mathrm{H}$ ). On the one hand, by (1), $2 b \sin \alpha>b$ and the vertical distance between $P$ and $s$ is at least $b$; hence $d(x, s)>b$ for any $x \in P$. On the other hand, the distance from any point of $P$ to the nearest vertex is less than $\frac{a}{2}+\frac{b}{2}$, which is less than $b$. Hence the property 


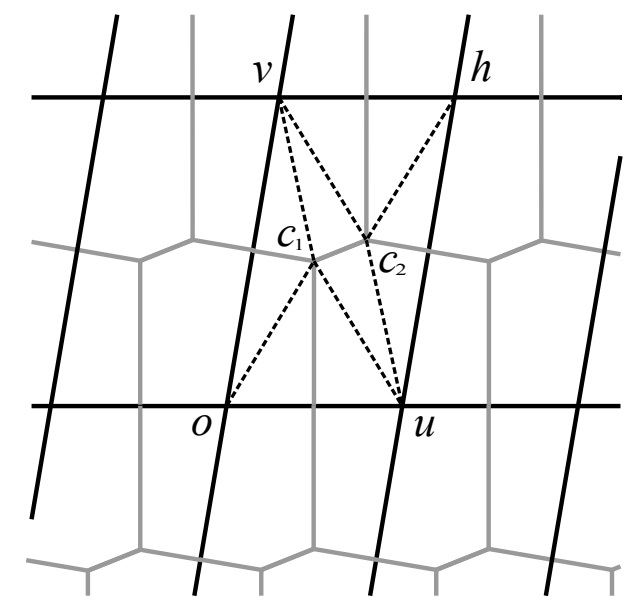

Figure 2: Voronoi diagram of the lattice $\mathbb{Z} u+\mathbb{Z} v$.

also holds for those vertices, and by symmetry, for $s=k u-k^{\prime} v, k^{\prime} \geq 2, k \in \mathbb{Z}$.

Now, it is easy to check that the vertices of the Voronoi diagram (gray in Figure 22 are the circumcenters $c_{1}$ and $c_{2}$ of $(o, u, v)$ and $(u, v, h)$. Obviously the farthest points from $p=\phi(o)=\phi(u)=\phi(v)=\phi(h)$ are $\phi\left(c_{1}\right)$ and $\phi\left(c_{2}\right)$, and the segments joining them to $p$ are the images by $\phi$ of the radii (dashed line in Figure 22.

\section{Flat Klein bottles}

Let $K_{a, b}$ be the Klein bottle obtained from an $a \times b$ rectangle by identifying the points of the $a$-long sides parallelly, and the points of the $b$-long sides by the symmetry with respect to the center of the rectangle, $a, b \in \mathbb{R}_{+}^{*}$. The direction of the $a$-long sides will be called horizontal, while the direction of the $b$-long sides will be called vertical. The horizontal and vertical geodesics of $K_{a, b}$ are closed. All horizontal geodesics have length $2 a$, excepting the one corresponding to the sides of the rectangle, and the one corresponding to the mid-height horizontal segment, which both have length $a$; we call them the main geodesics of $K_{a, b}$.

Theorem 3. Any flat Klein bottle is isometric to some $K_{a, b}$.

Proof. Let $K$ be a flat Klein bottle. Consider a closed curve $\gamma$ whose $\varepsilon$-strip is a Möbius strip, for some $\varepsilon>0$. Let $\gamma_{0}$ be a shortest closed curve homotopic to $\gamma ; \gamma_{0}$ has the same property. Moreover, $\gamma_{0}$ is a geodesic; denote by $a$ its length. For $\varepsilon>0$ small enough, an $\varepsilon$-strip of $\gamma_{0}$ is a flat Möbius strip, that is, an $a \times \varepsilon$ rectangle with the $\varepsilon$-long sides identified. The boundary of the strip is a closed geodesic of length $2 a$, say $\beta_{\varepsilon}: \mathbb{R} / 2 a \mathbb{Z} \rightarrow K$. Since $K$ has finite area, for greater $\varepsilon$, the strip must self overlap; let $b / 2$ be the greatest $\varepsilon$ such that it does not. There are distinct points $t_{0}, t_{1} \in \mathbb{R} / 2 a \mathbb{Z}$ such that $\beta_{b / 2}\left(t_{0}\right)=\beta_{b / 2}\left(t_{1}\right)$. 
Moreover

$$
\dot{\beta}_{b / 2}\left(t_{0}\right)= \pm \dot{\beta}_{b / 2}\left(t_{1}\right),
$$

for otherwise $\beta_{\varepsilon}$ would have self-intersected for lower $\varepsilon$. It follows that the lowest $\varepsilon$ such that $\beta_{\varepsilon}(t)$ has more that one preimage in $\mathbb{R} / 2 a \mathbb{Z}$ does not depend on $t$ and is $b / 2$. This defines an involution $\phi: \mathbb{R} / 2 a \mathbb{Z} \rightarrow \mathbb{R} / 2 a \mathbb{Z}$, without fixed points, such that $\beta_{b / 2}(t)=\beta_{b / 2}(\phi(t))$. Moreover, from (4), $\phi\left(t_{0}+s\right)=t_{1} \pm s$. Since $\phi$ has no fixed points, the minus case cannot occur. Since $\phi$ is an involution, $t_{1}-t_{0}=a$, and the proof is complete.

The circle $\mathbb{R} / a \mathbb{Z}$ acts isometrically on $K_{b}$ by horizontal translation. Other important isometries are the "reflections" with respect to the main geodesics, and the reflection with respect to a horizontal geodesic which exchanges the main geodesics (i.e., $\beta_{b / 2}$ in the above proof). It follows that any geometrical property attached to a point only depends on its distance $y \in[0, b / 4]$ to the nearest main geodesic.

Theorem 4. Let $p$ be a point of the flat Klein bottle $K_{a, b}$. Denote by $\sigma$ the union of its main geodesics and put $\lambda=2 d(p, \sigma) / b$. Note that $\lambda \in[0,1 / 2]$.

1. Assume $b<2 a$. Then $\# F_{p}=\# F_{p}^{4}=1$ if and only if $\lambda=0$; otherwise $\# F_{p}=\# F_{p}^{3}=2$.

2. Assume $b=2 a$. Then $\# F_{p}=\# F_{p}^{4}=1$ if and only if $\lambda=0$ or $\lambda=1 / 2$; otherwise $\# F_{p}=\# F_{p}^{3}=2$.

3. Assume $b>2 a$ and put $\lambda_{0}=\frac{1}{2}-\sqrt{\frac{1}{4}-\frac{a^{2}}{b^{2}}}$.

(a) If $\lambda=0$ then $\# F_{p}=\# F_{p}^{4}=1$.

(b) If $0<\lambda<\lambda_{0}$ then $\# F_{p}=\# F_{p}^{3}=2$.

(c) If $\lambda=\lambda_{0}$ then $\# F_{p}=\# F_{p}^{4}=1$.

(d) If $\lambda_{0}<\lambda<1 / 2$ then $\# F_{p}=\# F_{p}^{3}=1$.

(e) If $\lambda=1 / 2$ then $\# F_{p}=\# F_{p}^{3}=2$.

Proof. $K_{a, b}$ is isometric to the standard plane $\mathbb{R}^{2}$ quotiented by the group of affine isometries generated by the translation of vector $(0, b)$ and the glide reflection of vector $(0, a)$ whose axis is the $x$-axis. Let $\phi: \mathbb{R}^{2} \rightarrow K_{a, b}$ be the canonical surjection. The main geodesics (dot lines in Figure 3 ) are the image by $\phi$ of the lines of equations $y=n b / 2, n \in \mathbb{Z}$. By symmetry, one can assume without loss of generality that $p=\phi\left(p_{0}\right)$ with $p_{0}=(0,-\xi), \xi \in[0, b / 4]$ and set $\lambda=2 \xi / b$. Put $S_{0} \stackrel{\text { def }}{=} p_{0}+(2 a, 0) \mathbb{Z}+(0, b) \mathbb{Z} \subset \mathbb{R}^{2}$ and $S_{1}=S_{0}+(a,-2 \xi)$ (black and gray dots respectively in Figure 3 . We have

$$
\phi^{-1}(p)=S_{0} \cup S_{1} \stackrel{\text { def }}{=} S,
$$

and the cut locus of $p$ is the image under $\phi$ of the Voronoi diagram of $S$. 
If $\xi=0$, the Voronoi diagram is a regular rectangle tiling of the plane, and the conclusion follows immediately. So we may assume, from now on, that $\xi$ is positive.

Line segments from $x \in S_{0}$ to $x+( \pm a, b-2 \xi)$ and from $x$ to $x+( \pm a,-2 \xi)$ draw a regular tiling of the plane whose vertices are the points of $S$ and the tile is kite shaped.

We claim that the restriction to a kite $K$ of the Voronoi diagram of $S$ is the same as the Voronoi diagram of the four vertices of $K$. For this, it is sufficient to prove that for any point $x \in K$ and any $s \in S$ which is not a vertex of $K$, there is a vertex $w$ of $K$ such that $d(s, x)>d(v, x)$. Choose, for instance, the kite $K$ whose vertices are $p_{0}, v \stackrel{\text { def }}{=}(0, b-\xi)$ and $h^{ \pm}=( \pm a, \xi)$. Let $u=(0, \xi)$ be the intersection of its diagonals. If $s$ belongs to the positive half of the ordinates axis, then we can choose $w=v$, for the angle $\measuredangle x v^{+} s$ is obtuse. Similarly, one can chose $v=p_{0}$ if $s$ belongs to the negative part of the same axis and $v=h^{ \pm}$if $x$ belongs to the line $h^{-} h^{+}$. Now assume that $s$ belongs to the open quarter of plane bounded by half-lines starting at $u$ through $h^{+}$and $v$. With $q \stackrel{\text { def }}{=}(a, b-\xi)$, it is easy to see that on the one hand $\min \left(d(x, v), d\left(x, h^{+}\right)\right) \leq d(x, q)$, and on the other hand $\measuredangle x q s>\frac{\pi}{2}$, whence

$$
\min \left(d(x, v), d\left(x, h^{+}\right)\right)<d(x, s) .
$$

The cases of the three other quarters of plane are totally similar, so the claim is proved.

Denote by $\omega(u, v, w)$ the circumcenter of the triangle determined by $u, v$, $w$, where $w$ is a variable point in the plane. Put $\Delta \stackrel{\text { def }}{=} a^{2}-b^{2} \lambda(1-\lambda)$. By a straightforward computation, we have

$$
\begin{aligned}
c^{ \pm} \stackrel{\text { def }}{=} \omega\left(p_{0}, v, h^{ \pm}\right) & =\left( \pm \frac{\Delta}{2 a}, \frac{b}{2}(1-\lambda)\right), \\
c_{0} \stackrel{\text { def }}{=} \omega\left(p_{0}, h^{+}, h^{-}\right) & =\left(0, \frac{a^{2}}{2 b \lambda}\right), \\
c_{1} & \stackrel{\text { def }}{=} \omega\left(v, h^{-}, h^{+}\right)=\left(0, \frac{b^{2}(1-\lambda)^{2}-\Delta}{2 b(1-\lambda)}\right) .
\end{aligned}
$$

It follows that

$$
\begin{aligned}
d\left(c_{0}, v\right)^{2}-d\left(c_{0}, p_{0}\right)^{2} & =-\frac{\Delta}{\lambda}, \\
d\left(c^{+}, h^{-}\right)^{2}-d\left(c^{+}, p_{0}\right)^{2} & =2 \Delta, \\
d\left(c_{1}, v\right)^{2}-d\left(c_{1}, p_{0}\right)^{2} & =\frac{\Delta}{1-\lambda} .
\end{aligned}
$$

We have to discuss three cases, according the sign of $\Delta$.

Case $\Delta>0$. Note that this is the only possible case if $b \leq 2 a$. From (5),

$$
d\left(c_{0}, v\right)<d\left(c_{0}, p_{0}\right)=d\left(c_{0}, h^{+}\right)=d\left(c_{0}, h^{-}\right),
$$




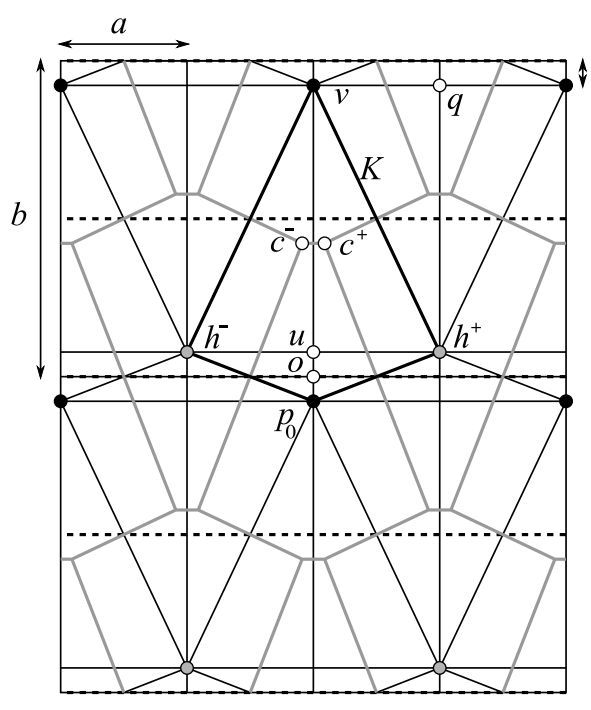

$\Delta>0$

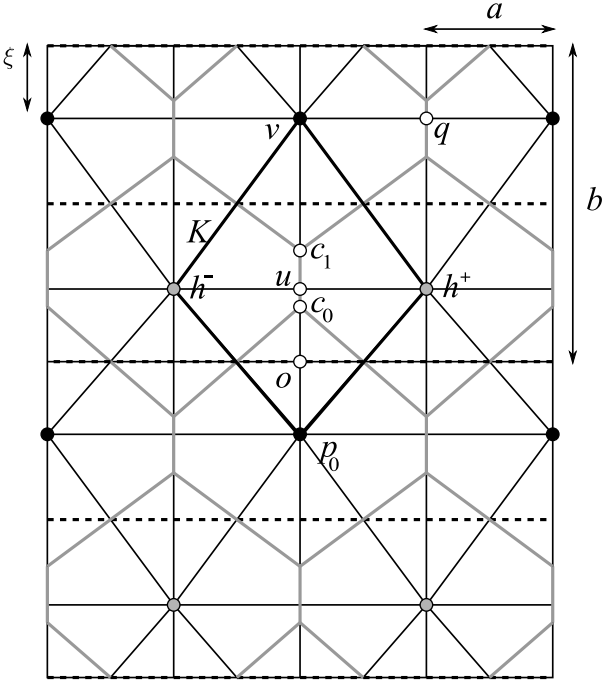

$\Delta<0$

Figure 3: Tiling (black lines) of the plane generated by a flat Klein bottle $K_{a, b}$ with the Voronoi diagram (gray lines) of the preimage of a point $p$.

whence $c_{0}$ belongs to the open Voronoi cell of $v$ and, consequently, is not a vertex of the Voronoi diagram. Similarly, by (7),

$$
d\left(c_{1}, p_{0}\right)<d\left(c_{1}, v\right)=d\left(c_{1}, h^{+}\right)=d\left(c_{1}, h^{-}\right),
$$

so $c_{1}$ belongs to the open Voronoi cell of $o$. It follows that the vertices of the restriction to $K$ of the Voronoi diagram of $S$ are precisely $c^{+}$and $c^{-}$. The whole Voronoi diagram is shown in Figure 3 as a gray line; $\phi\left(c^{+}\right)$and $\phi\left(c^{-}\right)$ are the only points of ramification of the cut-locus of $p$. Due to the vertical axis symmetry,

$$
d\left(\phi\left(c^{+}\right), p\right)=d\left(p_{0}, c^{+}\right)=d\left(p_{0}, c^{-}\right)=d\left(\phi\left(c^{-}\right), p\right),
$$

whence $\phi\left(c^{+}\right)$and $\phi\left(c^{-}\right)$are both farthest points from $p$. The points $c^{ \pm}$are of degree three in the Voronoi diagram, whence $\phi\left(c^{ \pm}\right) \in F_{p}^{3}$.

Case $\Delta<0$. By (6),

$$
d\left(c^{+}, h^{-}\right)<d\left(c^{+}, p_{0}\right)=d\left(c^{+}, h^{+}\right)=d\left(c^{+}, v\right),
$$

whence $c^{+}$does not belong to the cell of $h^{+}$, and consequently, it is not a vertex of the Voronoi diagram. The same argument holds for $c^{-}$. Moreover, by (5) and (7),

$$
\begin{aligned}
& d\left(c_{0}, v\right)>d\left(c_{0}, o\right)=d\left(c_{0}, h^{+}\right)=d\left(c_{0}, h^{-}\right), \\
& d\left(c_{1}, o\right)>d\left(c_{1}, v\right)=d\left(c_{1}, h^{+}\right)=d\left(c_{1}, h^{-}\right),
\end{aligned}
$$


whence $c_{0}$ and $c_{1}$ are the actual vertices of the Voronoi diagram. The whole Voronoi diagram is shown in Figure $3 . \phi\left(c^{+}\right)$and $\phi\left(c^{-}\right)$are the only points of ramification of the cut-locus of $p$. A simple computation shows that

$$
\begin{aligned}
d\left(p, \phi\left(c_{0}\right)\right)^{2}-d\left(p, \phi\left(c_{1}\right)\right)^{2} & =d\left(p_{0}, c_{0}\right)^{2}-d\left(v, c_{1}\right)^{2} \\
& =\frac{(1-2 \lambda) \Delta\left(a^{2}+b^{2}(1-\lambda) \lambda\right)}{4 b^{2}(1-\lambda)^{2} \lambda^{2}}
\end{aligned}
$$

is non-positive and vanishes if and only if $\lambda=1 / 2$. It follows that, for $\lambda<1 / 2$, $F_{p}=F_{p}^{3}=\left\{\phi\left(c_{1}\right)\right\}$, and for $\lambda=1 / 2, F_{p}=F_{p}^{3}=\left\{\phi\left(c_{0}\right), \phi\left(c_{1}\right)\right\}$.

Case $\Delta=0$. This case occurs only if $b \geq 2 a$ and corresponds to a single value of $\lambda \in] 0,1 / 2]$, namely

$$
\lambda=\frac{1}{2}-\sqrt{\frac{1}{4}-\frac{a^{2}}{b^{2}}} .
$$

In this case $c^{+}=c^{-}=c_{0}=c_{1}$. Hence $F_{p}^{4}=F_{p}=\left\{\phi\left(c_{0}\right)\right\}$.

\section{References}

[1] Y. Burago, M. Gromov and G. Perel'man, A. D. Alexandrov spaces with curvature bounded below, Russ. Math. Surv. 47 (1992), 1-58 (English. Russian original)

[2] H. T. Croft, K. J. Falconer and R. K. Guy, Unsolved Problems in Geometry. Springer-Verlag, New York, 1991

[3] J.-I. Itoh, J. Rouyer and C. Vîlcu, Antipodal convex hypersurfaces, Indag. Math., New Ser. 19 (2008), 411-426

[4] J.-I. Itoh and C. Vîlcu, What do cylinders look like?, J. Geom. 95 (2009), $41-48$

[5] Y. G. Nikonorov, Y. V. Nikonorova, The Intrinsic Diameter of the Surface of a Parallelepiped, Discrete \& Comput. Geometry 40 2008, 504-527

[6] J. Rouyer, Antipodes sur un tétraèdre régulier, J. Geom. 77 (2003), 152-170

[7] J. Rouyer, Steinhaus conditions for convex polyhedra, in: Convexity and Discrete Geometry Including Graph Theory, K. Adiprasito et al. (Eds.), Springer Proc. in Mathematics and Statistics 148 (2016), 77-84

[8] J. Rouyer and C. Vîlcu, The connected components of the space of Alexandrov surfaces, in D. Ibadula and W. Veys (eds.), Experimental and Theoretical Methods in Algebra, Geometry and Topology, Springer Proc. in Mathematics and Statistics 96 (2014), 249-254

[9] J. Rouyer and C. Vîlcu, Farthest points on most Alexandrov surfaces, Adv. Geom., to appear; arXiv:1412.1465 [math.MG] 
[10] K. Shiohama, An introduction to the geometry of Alexandrov spaces, Lecture Notes Series, Seoul National University, 8 (1992)

[11] K. Shiohama and M. Tanaka, Cut loci and distance spheres on Alexandrov surfaces, Actes de la Table Ronde de Géométrie Différentielle (Luminy, 1992), Sém. Congr., vol. 1, Soc. Math. France, Paris, 1996, 531-559

[12] C. Vîlcu, On two conjectures of Steinhaus, Geom. Dedicata 79 (2000), 267275

[13] C. Vîlcu, Properties of the farthest point mapping on convex surfaces, Rev. Roum. Math. Pures Appl. 51 (2006), 125-134

[14] C. Vîlcu, T. Zamfirescu, Symmetry and the farthest point mapping on convex surfaces, Adv. Geom. 6 (2006), 345-353

[15] C. Vîlcu and T. Zamfirescu, Multiple farthest points on Alexandrov surfaces, Adv. Geom. 7 (2007), 83-100

[16] T. Zamfirescu, On some questions about convex surfaces. Math. Nach. 172 (1995), 313-324

[17] T. Zamfirescu, Points joined by three shortest paths on convex surfaces, Proc. Am. Math. Soc. 123 (1995), 3513-3518

[18] T. Zamfirescu, Extreme points of the distance function on convex surfaces, Trans. Amer. Math. Soc. 350 (1998), 1395-1406 